pag

Business School

WORKING PAPER SERIES

Working Paper

2014-158
Multiple Interior Steady States in the Ramsey Model with Elastic Labor Supply

Takashi Kamihigashi

http://www.ipag.fr/fr/accueil/la-recherche/publications-WP.html

IPAG Business School

184, Boulevard Saint-Germain

75006 Paris

France 


\title{
Multiple Interior Steady States in the Ramsey Model with Elastic Labor Supply*
}

\author{
Takashi Kamihigashi ${ }^{\dagger \ddagger}$
}

March 18, 2014

\begin{abstract}
In this paper we show that multiple interior steady states are possible in the Ramsey model with elastic labor supply. In particular we establish the following three results: (i) for any discount factor and production function, there is a utility function such that a continuum of interior steady states exist; (ii) the number of interior steady states can also be any finite number; and (iii) for any discount factor and production function, there is a utility function such that there is no interior steady state. Some numerical examples are provided.
\end{abstract}

*Financial support from the Japan Society for the Promotion of Science is gratefully acknowledged.

${ }^{\dagger}$ RIEB, Kobe University, Rokkodai, Nada, Kobe 657-8501 Japan.

Email: tkamihig@rieb.kobe-u.ac.jp

${ }^{\ddagger}$ IPAG Business School, 184 Bd Saint Germain, 75006 Paris, France. 


\section{Introduction}

One-sector neoclassical optimal growth models have been used extensively to address two major issues in macroeconomics: long-run growth and short-run fluctuations. A special case of this class of models with inelastic labor supply is known as the Ramsey model, and one of its important properties is that all optimal paths converge to a unique steady state. There is a large empirical literature on cross-country implications of this convergence property (e.g., Barro and Sala-i-Martin, 2004). Stochastic versions of the Ramsey model with elastic labor supply are known as RBC (real business cycle) models (e.g., King et al., 1988; Christiano and Eichenbaum, 1992), which are in turn considered to be prototype DSGE (dynamic stochastic general equilibrium) models. These models are often solved by linear approximation around a steady state, which is typically assumed to be unique.

In this paper we study the possibility of multiple steady states in the Ramsey model with elastic labor supply. Despite the importance of this model, to our knowledge, there has been no systematic analysis of this problem in the literature though there are various parametric models whose steady states are solved explicitly!

The literature on multiple steady states in one-sector neoclassical growth models dates back to Diamond (1965), who considered an overlapping generations model; see Galor and Ryder (1989) for subsequent results. More closely related to this paper is the analysis of multiple steady states in the context of optimal growth, dating back to Kurz (1968). He showed that multiple steady states are possible if utility is a function of consumption and capital.

In this paper we show that multiple interior steady states are possible if utility is a function of consumption and leisure, and if leisure is not a normal good. In particular we establish the following three results: (i) for any discount rate and production function (satisfying standard conditions), there is a utility function such that a continuum of interior steady states exist; (ii) the number of interior steady states can also be any finite number;

\footnotetext{
${ }^{1}$ In the case of inelastic labor supply, the aforementioned convergence property (which implies the existence of a unique steady state) was established by Cass (1965) and Koopmans (1965) for the continuous time case, and by Brock and Mirman (1972, Section 2) for the discrete time case. Le Van et al. (2007) showed a convergence property for the case of elastic labor with heterogenous agents. Various convergence results on general optimal growth models are also available; see Yano $(1999,2012)$ and the references therein.
} 
and (iii) for any discount factor and production function, there is a utility function such that there is no interior steady state.

In a wider context, the connection between multiplicity of steady states and inferiority of goods is already well recognized; see, e.g., De Hek (1998) and Bond et al. (2012, 2014). In fact, De Hek's work is most closely related to this paper; he showed through numerical examples that multiple steady states and non-monotone dynamics are possible in a similar model. His model differs from ours in that current output is assumed to be a function of the levels of capital and labor chosen in the previous period ${ }^{2}$

The rest of the paper is organized as follows. In the next section we present the model. In Section 3 we show some preliminary results. In Section 4 we establish our main results. In Section 5 we offer some numerical examples. All proofs appear in the Appendix unless otherwise indicated.

\section{The Model}

Consider the following one-sector optimal growth model:

$$
\begin{array}{cl}
\max _{\left\{c_{t}, n_{t}, k_{t+1}\right\}_{t=0}^{\infty}} & \sum_{t=0}^{\infty} \beta^{t} u\left(c_{t}, n_{t}\right) \\
\text { s.t. } \forall t \geq 0, & c_{t}+k_{t+1}=f\left(k_{t}, n_{t}\right), \\
& 0 \leq n_{t} \leq 1, \\
& c_{t}, k_{t+1} \geq 0, \\
& k_{0}>0 \text { given, }
\end{array}
$$

where $c_{t}$ is consumption, $n_{t}$ is labor $\left(1-n_{t}\right.$ is leisure), $k_{t}$ is capital, $\beta \in(0,1)$ is the discount factor, $u$ is the utility function, and $f$ is the production function. We state our assumptions after introducing some definitions.

A feasible plan (from $\bar{k}_{0}$ ) is a set of paths $\left\{c_{t}, n_{t}, k_{t}\right\}_{t=0}^{\infty}$ satisfying 2.2 (2.4) (with $k_{0}=\bar{k}_{0}$ ). An interior plan is a feasible plan such that none of the inequality constraints in (2.3) and (2.4) is binding. An optimal plan is a feasible plan that solves the above maximization problem. An interior optimal plan is an optimal plan that is also an interior plan.

Throughout the paper, we maintain the following assumptions.

\footnotetext{
${ }^{2}$ In our notation, this means that the right-hand side of 2.2$)$ is $f\left(k_{t}, n_{t-1}\right)$. See Cai et al. (2013) for a stochastic version of this specification.
} 
Assumption 2.1. (f1) $f: \mathbb{R}_{+}^{2} \rightarrow \mathbb{R}_{+}$is $C^{1}$ on $\mathbb{R}_{++}^{2}$, concave, strictly concave in the first argument, and linearly homogeneous. (f2) For all $(k, n) \in \mathbb{R}_{++}^{2}$, we have

$$
f_{1}(k, n)>0, \quad f_{2}(k, n)>0 .
$$

(f3) There exists $\bar{k}>0$ such that

$$
f(\bar{k}, 1)=\bar{k} .
$$

(f4) $k_{0} \in(0, \bar{k}]$. (f5) There exists $(\tilde{k}, \tilde{n}) \in \mathbb{R}_{++}^{2}$ such that

$$
\beta f_{1}(\tilde{k}, \tilde{n})=1 .
$$

Since $f(k, 1)$ is strictly concave in $k$ by (f1), condition (f4) implies that for any feasible plan $\left\{c_{t}, n_{t}, k_{t}\right\}$ from $k_{0} \leq \bar{k}$ we have

$$
\forall t \in \mathbb{Z}_{+}, \quad c_{t}, k_{t} \leq \bar{k} .
$$

We restrict the domain of $u$ accordingly:

Assumption 2.2. (u1) There exists $\bar{c}>\bar{k}$ such that $u:[0, \bar{c}] \times[0,1] \rightarrow$ $\mathbb{R} \cup\{-\infty\}$ is $C^{1}$ on $(0, \bar{c}) \times(0,1)$ and strictly concave. (u2) For all $(c, n) \in$ $(0, \bar{c}) \times(0,1)$ we have

$$
u_{1}(c, n)>0, \quad u_{2}(c, n)<0 .
$$

It is easy to see that any interior optimal plan satisfies the the following conditions for all $t \in \mathbb{Z}_{+}$:

$$
\begin{array}{r}
-u_{1}\left(f\left(k_{t}, n_{t}\right)-k_{t+1}, n_{t}\right)+\beta u_{1}\left(f\left(k_{t+1}, n_{t+1}\right)-k_{t+2}, n_{t+1}\right) f_{1}\left(k_{t+1}, n_{t+1}\right)=0, \\
(2.11) \\
u_{1}\left(f\left(k_{t}, n_{t}\right)-k_{t+1}, n_{t}\right) f_{2}\left(k_{t}, n_{t}\right)+u_{2}\left(f\left(k_{t}, n_{t}\right)-k_{t+1}, n_{t}\right)=0 .
\end{array}
$$

Equation (2.11) is the Euler equation for $k_{t+1}$, and equation 2.12 is the first-order condition for $n_{t}$. The transversality condition is

$$
\lim _{t \uparrow \infty} \beta^{t} u_{1}\left(f\left(k_{t}, n_{t}\right)-k_{t+1}, n_{t}\right) k_{t+1}=0 .
$$

Since $u$ and $f$ are concave, 2.11 -2.13 are sufficient for optimality $3^{3}$

\footnotetext{
${ }^{3}$ See Kamihigashi (2005) for discussion on the transversality condition.
} 
We define an interior steady state as a pair $(k, n) \in(0, \bar{k}) \times(0,1)$ such that

$$
\begin{gathered}
\beta f_{1}(k, n)=1, \\
u_{1}(f(k, n)-k, n) f_{2}(k, n)+u_{2}(f(k, n)-k, n)=0, \\
f(k, n)-k>0 .
\end{gathered}
$$

For any interior steady state $(k, n)$, a feasible plan $\left\{c_{t}, n_{t}, k_{t}\right\}$ with $k_{t}=k$ and $n_{t}=n$ for all $t \in \mathbb{Z}_{+}$is an interior optimal plan since it satisfies (2.11)-(2.13) with $c_{t}=f(k, n)-k>0$.

\section{Preliminary Results}

In this section we define useful constants, and provide simple characterizations of interior steady states.

Lemma 3.1. There exits a unique constant $\gamma>0$ such that for any $(k, n) \in$ $\mathbb{R}_{++}^{2}$ we have

$$
\beta f_{1}(k, n)=1 \Longleftrightarrow k=\gamma n .
$$

Lemma 3.1 implies that any interior steady state $(k, n)$ satisfies $k=$ $\gamma n$; i.e., the capital-labor ratio is constant across all possible interior steady states. This allows us to focus on levels of labor in characterizing interior steady states. We say that $n \in(0,1)$ is a steady state level of labor if $(k, n)$ with $k=\gamma n$ is an interior steady state.

We further define the following constants:

$$
\begin{aligned}
\mu & =f_{2}(\gamma, 1)>0, \\
\lambda & =f(\gamma, 1)-\gamma \\
\rho & =\frac{1}{\beta}-1>0 .
\end{aligned}
$$

The inequality in 3.2 is immediate from (2.6). By linear homogeneity, for all $n>0$ we have

$$
\begin{aligned}
\mu & =f_{2}(\gamma n, n), \\
\lambda n & =f(\gamma n, n)-\gamma n .
\end{aligned}
$$


Thus $\mu$ and $\lambda$ are the marginal product of labor and the consumption-labor ratio, respectively, in any interior steady state. Differentiating (3.6) with respect to $n$ and using (3.1) and (3.6), we obtain

$$
\lambda=\mu+\rho \gamma>0 \text {. }
$$

The following result shows that the steady state levels of labor are characterized by a single equation.

Lemma 3.2. Let $n \in(0,1)$. Then $n$ is a steady state level of labor if and only if

$$
g(n) \equiv u_{1}(\lambda n, n) \mu+u_{2}(\lambda n, n)=0 .
$$

The proofs of the following two corollaris are straightforward and thus omitted.

Corollary 3.1. There exists an interior steady state if (3.9) or (3.10) below holds:

$$
\begin{aligned}
& \lim _{n \downarrow 0} g(n)<0<\lim _{n \uparrow 1} g(n), \\
& \lim _{n \downarrow 0} g(n)>0>\lim _{n \uparrow 1} g(n) .
\end{aligned}
$$

Corollary 3.2. Suppose that there exist $C^{1}$ functions $v:(0, \bar{c}) \rightarrow \mathbb{R} \cup\{\infty\}$ and $w:(0,1) \rightarrow \mathbb{R} \cup\{-\infty\}$ such that

$$
u(c, n)=v(c)+w(n) .
$$

Then there can be at most one interior steady state.

To obtain another useful characterization of interior steady states, consider the following maximization problem:

$$
\max _{c \geq 0, n \in[0,1]} u(c, n) \quad \text { s.t. } c-\mu n=y,
$$

where $y \in(0, \bar{k})$. Since $u$ is strictly concave, this problem has a unique solution, which we denote by $\left(c^{*}(y), n^{*}(y)\right)$. Provided that $n \in(0,1)$, we have $n=n^{*}(y)$ if and only if

$$
u_{1}(y+\mu n, n) \mu+u_{2}(y+\mu n, n)=0 .
$$

Exploring the similarity between (3.8) and (3.13), we obtain the following:

$$
{ }^{4} \lambda=f_{1}(\gamma n, n) \gamma+f_{2}(\gamma n, n)-\gamma=\gamma / \beta+\mu-\gamma=\rho \gamma+\mu .
$$


Lemma 3.3. Let $n \in(0,1)$. Then $n$ is a steady state level of labor if and only if

$$
n=n^{*}(\rho \gamma n) \text {. }
$$

Since $\mu$ is the marginal product of labor in any interior steady state, $\mu$ can be interpreted as a steady state wage rate. Hence the constraint in (3.12) can be viewed as a budget constraint under this wage rate given non-labor income $y$. We say that consumption is a normal (inferior) good if $c^{*}(y)$ is strictly increasing (decreasing) in $y$ whenever $n^{*}(y) \in(0,1)$. We say that leisure is a normal (inferior) good if $n^{*}(y)$ is strictly decreasing (increasing) in $y$ whenever $n^{*}(y) \in(0,1)$.

\section{Main Results}

The properties of consumption and leisure defined above are closely related to the possibility of multiple interior steady states, as we now see:

Proposition 4.1. If leisure is a normal good, then there can be at most one interior steady state.

If consumption is an inferior good, then leisure must be a normal good. Thus the following result is immediate from Proposition 4.1.

Corollary 4.1. If consumption is an inferior good, then there can be at most one interior steady state.

Proposition 4.1 implies that there can be multiple interior steady states only if leisure is not a normal good. The following result shows that there can even be a continuum of interior steady states.

Proposition 4.2. For any discount factor $\beta \in(0,1)$ and production function $f$ satisfying Assumption 2.1, there exists a utility function u satisfying Assumption 2.2 such that any $n \in(0,1)$ is a steady state level of labor.

The number of interior steady states can also be any finite number:

Proposition 4.3. Let $m \in \mathbb{N}$ and $0<\eta_{1}<\eta_{2}<\cdots<\eta_{m}<1$. Then for any discount factor $\beta \in(0,1)$ and production function $f$ satisfying Assumption 2.1. there exists a utility function $u$ satisfying Assumption 2.2 such that $n \in$ $(0,1)$ is a steady state level of labor if and only if $n \in\left\{\eta_{1}, \ldots, \eta_{m}\right\}$. 
Finally, it is even possible that no interior steady state exists:

Proposition 4.4. For any discount factor $\beta \in(0,1)$ and production function $f$ satisfying Assumptions 2.1, there exists a utility function u satisfying Assumptions 2.2 such that there exists no interior steady state.

\section{$5 \quad$ Numerical Examples}

In this section we provide some numerical examples to illustrate our results. We assume that $u$ is bounded below, so that we can rely on dynamic programming without technical concerns. The Bellman equation for the maximization problem (2.1)-2.5) can be written as

$$
v(k)=\max _{c, n, x}\{u(c, n)+\beta v(x): c+x=f(k, n), n \in[0,1], c, x \geq 0\},
$$

where $k \in[0, \bar{k}]$ and $v:[0, \bar{k}] \rightarrow \mathbb{R}$ is the value function. We fix $f$ and $\beta$ as follows:

$$
f(k, n)=k^{\alpha} n^{1-\alpha}, \quad \alpha=0.4, \quad \beta=0.95 .
$$

Under this specification, we have

$$
\gamma=(\alpha \beta)^{1 /(1-\alpha)} \approx 0.245, \mu=(1-\alpha) \gamma^{\alpha} \approx 0.172, \lambda \approx 0.185, \bar{k}=1 .
$$

In what follows, the value function and the associated policy functions are computed for three different utility functions $5^{5}$ Although we can use $g(n)$ in (3.8) to compute interior steady states, we wish to confirm our results by using a method that does not use $g(n)$ as well as to understand other aspects of the model.

The first utility function that we consider is

$$
u(c, n)=c^{0.4}-0.2 n^{1.2} .
$$

Figure 1(a) depicts this utility function as a function of consumption and leisure $(1-n)$. By Corollary 3.2, there can be at most one interior steady state under (5.4). This is confirmed by Figure 1(c), which shows that there is a unique interior steady state.

\footnotetext{
${ }^{5}$ All of these functions are computed by modified policy iteration with 10,000 equally spaced grid points.
} 


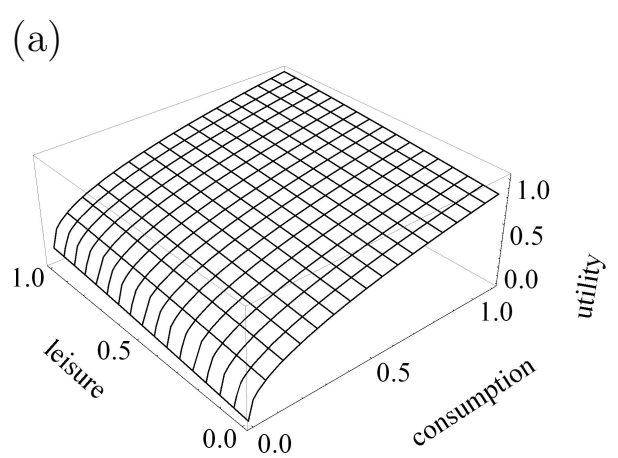

(b)

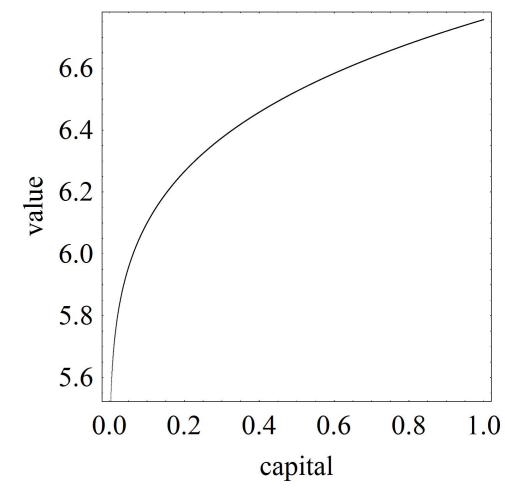

(d)

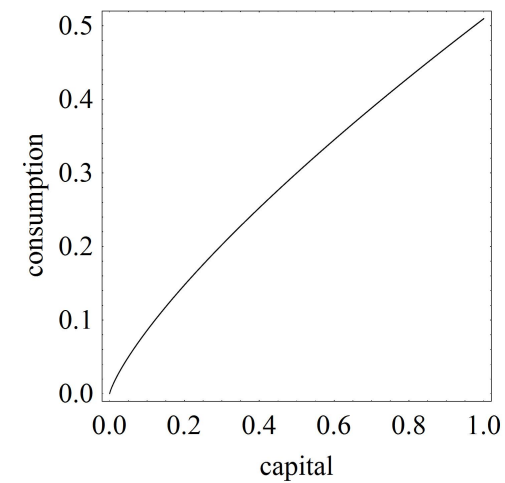

(c)

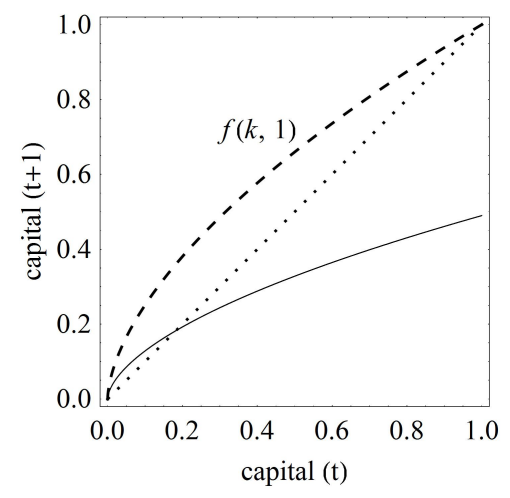

(e)

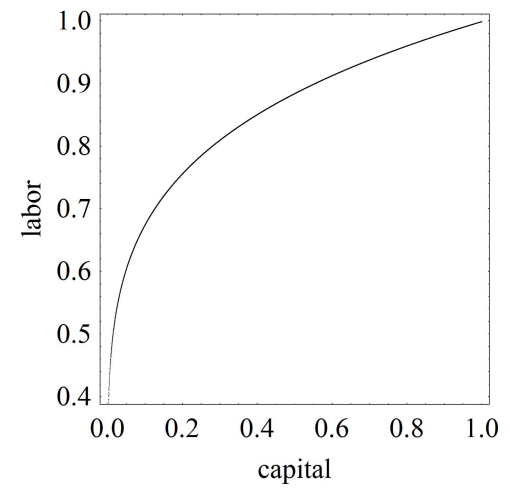

Figure 1: Functions computed under (5.4): (a) utility function $u(c, 1-l)$; (b) value function $v(k)$; and optimal policies for (c) capital $\left(k_{t+1}\right)$, (d) consumption $\left(c_{t}\right)$, and (e) labor $\left(n_{t}\right)$ as functions of current captial $k_{t}$ 
The second utility function that we consider is

$$
u(c, n)=\left[-\frac{(c-\sigma)^{2}}{2}+\nu\left(c-\frac{\sigma \mu}{\nu}\right) n-\frac{\tau^{2}}{2} n^{2}-\zeta\right]^{1 / 2},
$$

where

$$
\tau=\frac{\mu+\lambda}{2} \approx 0.179, \quad \nu=\frac{\tau^{2}+\lambda \mu}{\lambda+\mu} \approx 0.178, \quad \sigma=\frac{1.2 \bar{k} \nu}{\mu} \approx 1.244,
$$

and $\zeta$ is chosen so that $u(0,1)=0$. This function satisfies Assumption 2.2, as can be seen from Figure 2(a). The utility function constructed in the proof of Proposition 4.2 is the expression inside the square brackets in (5.5). As in the proof, the parameter values are chosen so that any $n \in(0,1)$ solves (3.8); i.e., any $n \in(0,1)$ is a steady state level of labor. This is confirmed by Figure 2(c),(e)

The last utility function that we consider is

$$
u(c, n)=\left[-\frac{(c-\sigma)^{2}}{2}+\nu\left(c-\frac{\sigma \mu}{\nu}\right)^{*} n-\frac{\tau^{2}}{2} n^{2}-\zeta\right]^{1 / 2}-\epsilon(n-1),
$$

where $\epsilon=0.01$. This is a perturbed version of (5.5); all parameter values are as above. Since (3.8) holds for all $n \in(0,1)$ under $(5.5)$, there is no $n \in(0,1)$ satisfying (3.8) under (5.7); i.e., there is no interior steady state. This is confirmed by Figure 3(c).

\section{Appendix A Proofs}

\section{A.1 Proof of Lemma 3.1}

Let $\gamma=\tilde{k} / \tilde{n}$, where $\tilde{k}$ and $\tilde{n}$ are given by Assumption 2.1. By linear homogeneity we have

$$
\forall n>0, \quad \beta f_{1}(\gamma n, n)=1 .
$$

We have shown the " $\Leftarrow$ " implication in 3.1 .

To verify the reverse implication, let $\left(k^{\prime}, n^{\prime}\right) \gg 0$ be such that $\beta f_{1}\left(k^{\prime}, n^{\prime}\right)=$ 1. Let $\gamma^{\prime}=k^{\prime} / n^{\prime}$. To show that $\gamma^{\prime}=\gamma$, define $m(\tilde{\gamma})=f_{1}\left(\tilde{\gamma} n^{\prime}, n^{\prime}\right)$ for $\tilde{\gamma}>0$. Then $m\left(\gamma^{\prime}\right)=f_{1}\left(k^{\prime}, n^{\prime}\right)=1 / \beta=m(\gamma)$, where the last equality holds by (A.1). Since $f$ is strictly concave in the first argument, $m(\cdot)$ is strictly decreasing. Hence $\gamma^{\prime}=\gamma$. 


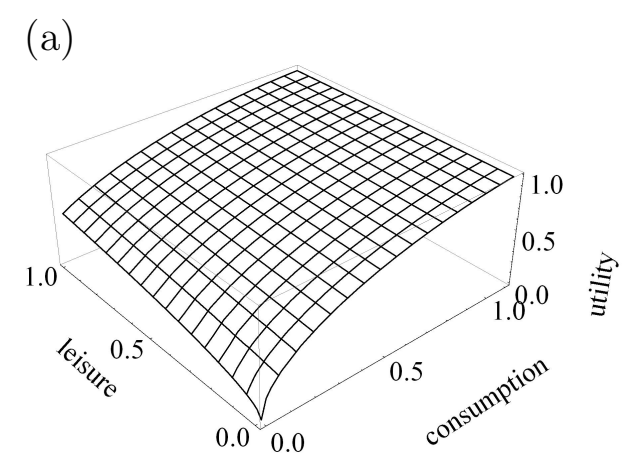

(b)

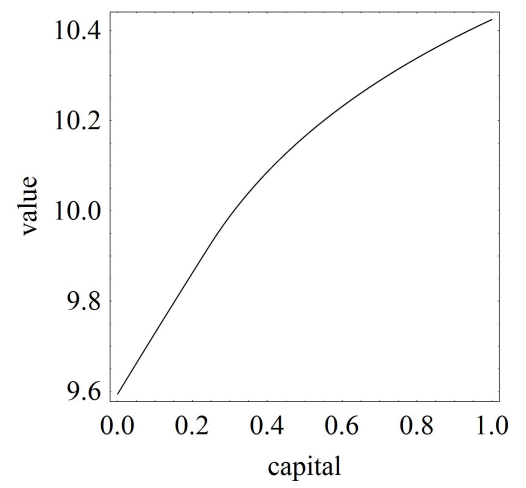

(d)

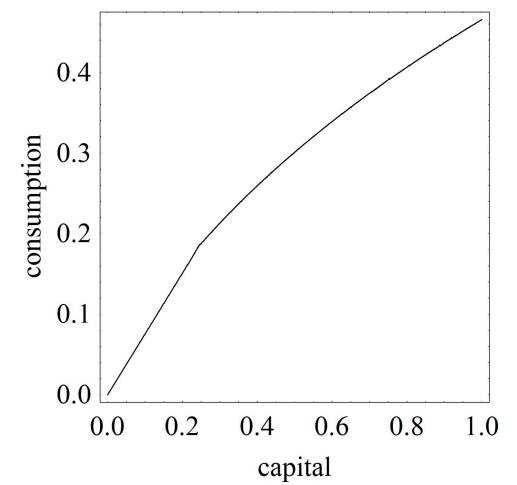

(c)

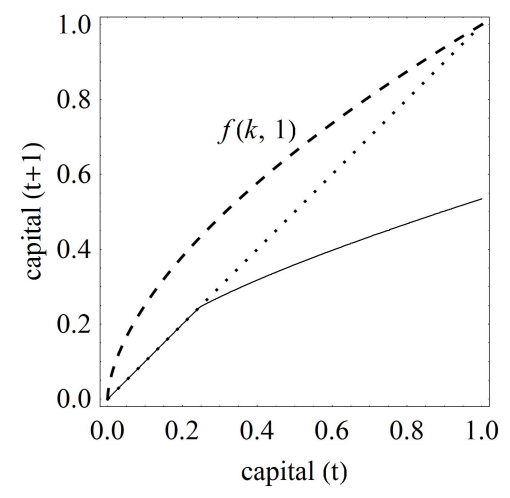

(e)

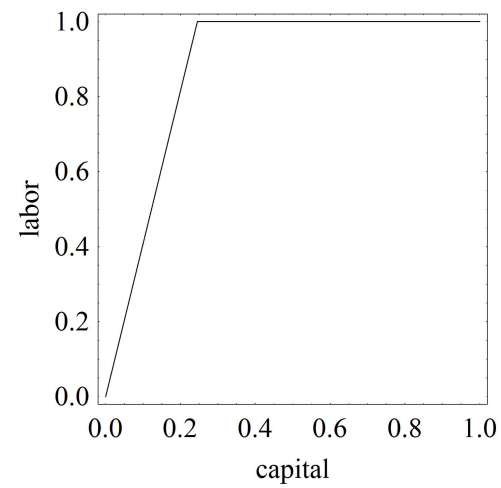

Figure 2: Functions computed under (5.5): (a) utility function $u(c, 1-l)$; (b) value function $v(k)$; and optimal policies for (c) capital $\left(k_{t+1}\right)$, (d) consumption $\left(c_{t}\right)$, and (e) labor $\left(n_{t}\right)$ as functions of current captial $k_{t}$ 


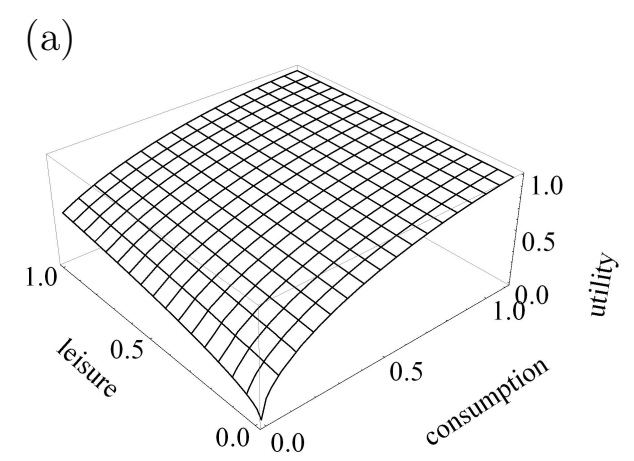

(b)

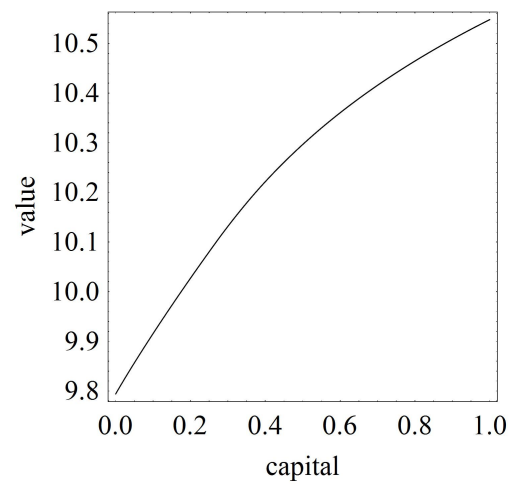

(d)

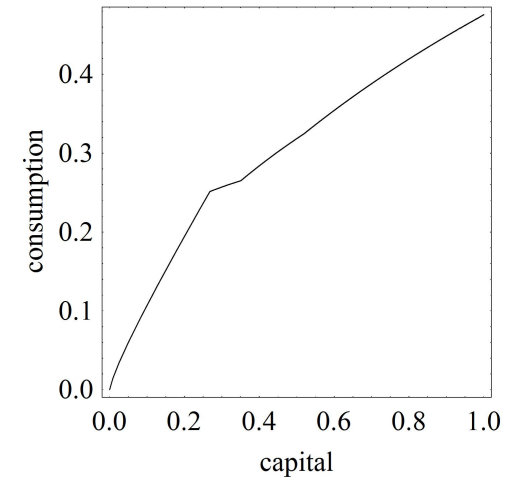

(c)

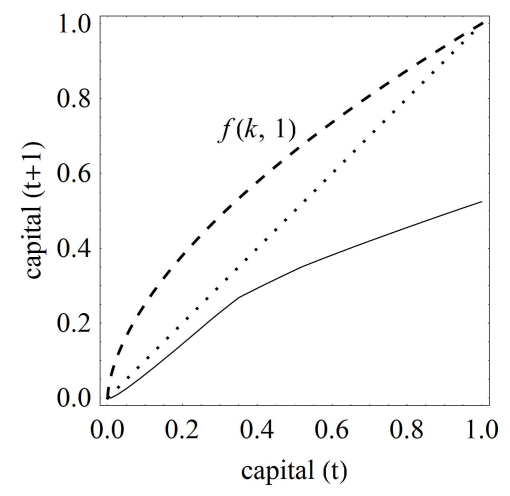

(e)

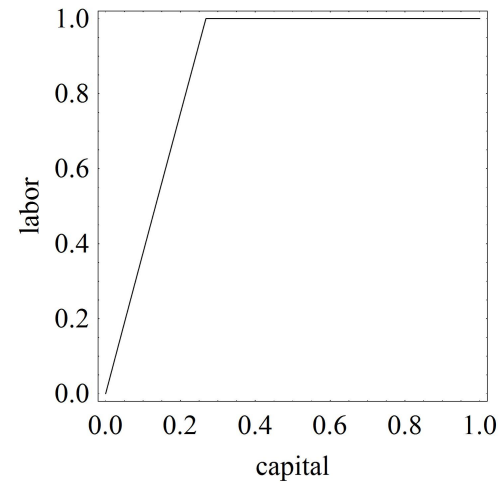

Figure 3: Functions computed under (5.7): (a) utility function $u(c, 1-l)$; (b) value function $v(k)$; and optimal policies for (c) capital $\left(k_{t+1}\right)$, (d) consumption $\left(c_{t}\right)$, and (e) labor $\left(n_{t}\right)$ as functions of current captial $k_{t}$ 


\section{A.2 Proof of Lemma 3.2}

Let $n \in(0,1)$ satisfy 3.8$)$. Let $k=\gamma n>0$. Then 2.14 holds by Lemma 3.1. By 3.6 we have

$$
f(k, n)-k=\lambda n>0 .
$$

Thus (2.16) holds. Since $f(k, 1)>f(k, n)>k$ by A.2), we have $k<\bar{k}$. We obtain (2.15) from (A.2) and (3.5). Hence $n$ is a steady state level of labor.

Conversely, let $(k, n) \in(0, k) \times(0,1)$ satisfy $2.14-2.16)$. Then $k=\gamma n$ by Lemma 3.1. Thus (A.2) holds. Substituting (A.2) and (3.5) into (2.15), we obtain 3.8 .

\section{A.3 Proof of Lemma 3.3}

Let $n \in(0,1)$. Suppose that $n=n^{*}(\rho \gamma n)$. It follows from 3.13 that

$$
u_{1}(\rho \gamma n+\mu n, n) \mu+u_{2}(\rho \gamma n+\mu n, n)=0 .
$$

Since $\rho \gamma+\mu=\lambda$ by (3.7), we obtain (3.8). Conversely, assume (3.8). Substituting (3.7) into (3.8), we obtain (A.3), which implies that $n=n^{*}(\rho \gamma n)$.

\section{A.4 Proof of Proposition 4.1}

Suppose that leisure is a normal good. Then $n^{*}(\cdot)$ is strictly decreasing. Hence there can be at most one solution $n$ to (3.14). Thus by Lemma 3.3. there can be at most one interior steady state.

\section{A.5 Proof of Proposition 4.2}

Define $\phi: \mathbb{R}^{2} \rightarrow \mathbb{R}$ by

$$
\phi(c, n)=-\frac{1}{2}(c-\sigma)^{2}+\nu\left(c-\frac{\sigma \mu}{\nu}\right) n-\frac{\tau^{2}}{2} n^{2},
$$

where $\sigma, \tau>0$ are parameters and $\nu$ is defined by

$$
\nu=\frac{\tau^{2}+\lambda \mu}{\lambda+\mu} .
$$

For all $c, n \in \mathbb{R}$ we have

$$
\phi_{1}(c, n)=(\sigma-c)+\nu n, \quad \phi_{2}(c, n)=\nu\left(c-\frac{\sigma \mu}{\nu}\right)-\tau^{2} n .
$$


Direct computation shows that for all $n \in \mathbb{R}$ we have ${ }^{6}$

$$
\phi_{1}(\lambda n, n) \mu+\phi_{2}(\lambda n, n)=0 .
$$

It thus follows by Lemma 3.2 that if $u=\phi$ on $[0, \bar{c}] \times[0,1]$ for some $\bar{c}>\bar{k}$, then any $n \in(0,1)$ is a steady state level of labor. Hence it suffices to show that $\phi$ satisfies Assumption 2.2 on $[0, \bar{c}] \times[0,1]$ for some $\sigma, \tau>0$ and $\bar{c}>\bar{k}$. To this end we need two lemmas:

Lemma A.1. Let

$$
\hat{c}=\frac{\sigma \mu}{\nu}
$$

Then

$$
\forall(c, n) \in[0, \hat{c}) \times[0,1], \quad \phi_{1}(c, n)>0, \quad \phi_{2}(c, n)<0 .
$$

Proof. Let $(c, n) \in[0, \hat{c}) \times[0,1]$. Since $\hat{c}<\sigma$, we have $\phi_{1}(c, n)>0$ by (A.6). Note that

$$
\phi_{2}(c, n)<\nu\left(\hat{c}-\frac{\sigma \mu}{\nu}\right)-\tau^{2} n=-\tau^{2} n<0 .
$$

This completes the proof.

Lemma A.2. $\phi$ is strictly concave if and only if

$$
\mu<\tau<\lambda
$$

Proof. Note from A.6 that the Hessian matrix of $\phi$ at any $(c, n)$ is

$$
\left[\begin{array}{ll}
\phi_{11} & \phi_{12} \\
\phi_{21} & \phi_{22}
\end{array}\right]=\left[\begin{array}{cc}
-1 & \nu \\
\nu & -\tau^{2}
\end{array}\right] .
$$

Thus $\phi$ is strictly concave if and only if $\nu^{2}<\tau^{2}$. Since $\nu, \tau>0$, it follows that $\phi$ is strictly concave if and only if $\nu<\tau$. Recalling A.5, we see that $\nu<\tau \Leftrightarrow \tau^{2}+\lambda \mu<(\lambda+\mu) \tau \Leftrightarrow \tau^{2}-(\lambda+\mu) \tau+\lambda \mu<0 \Leftrightarrow(\tau-\lambda)(\tau-\mu)<0$. The last inequality is equivalent to (A.11) since $\mu<\lambda$ by (3.7).

To complete the proof of Proposition 4.2, fix $\tau \in(\mu, \lambda)$ and $\bar{c}>\bar{k}$. Let $\sigma>0$ be such that $\hat{c}>\bar{c}$, where $\hat{c}$ and $\nu$ are given by (A.8) and (A.5), respectively. Suppose that $u(c, n)=\phi(c, n)$ for all $(c, n) \in[0, \bar{c}] \times[0,1]$. Then Lemmas A.1 and A.2 imply Assumption 2.2. Thus by A.7 and Lemma 3.2 , any $n \in(0,1)$ is a steady state level of labor.

\footnotetext{
${ }^{6} \phi_{1}(\lambda n, n) \mu+\phi_{2}(\lambda n, n)=[(\sigma-\lambda n)+\nu n] \mu+\nu(\lambda n-\sigma \mu / \nu)-\tau^{2} n=[\nu(\mu+\lambda)-(\lambda \mu+$ $\left.\left.\tau^{2}\right)\right] n+\sigma \mu-\sigma \mu=0$.
} 


\section{A.6 Proof of Proposition 4.3}

Let $m \in \mathbb{N}$ and $0<\eta_{1}<\eta_{2}<\cdots<\eta_{m}<1$. It suffices to show that there exists a utility function $u$ satisfying Assumption 2.2 such that $n \in(0,1)$ satisfies (3.8) if and only if $n \in\left\{\eta_{1}, \ldots, \eta_{m}\right\}$. To this end, let $J=\{1, \ldots, m\}$, and define $h: \mathbb{R}_{+} \rightarrow \mathbb{R}$ by

$$
h(n)=\int_{0}^{n} \prod_{j \in J}\left(x-\eta_{j}\right) d x .
$$

Since $h^{\prime}(n)=\prod_{j \in J}\left(n-\eta_{j}\right)$, we have $h^{\prime}(n)=0$ if and only if $n \in\left\{\eta_{1}, \ldots, \eta_{m}\right\}$.

Let $\bar{c}$ and $\phi$ be as in the proof of Proposition 4.2. Let $\epsilon \in \mathbb{R}$. Define $u(c, n)=\phi(c, n)+\epsilon h(n)$ for $(c, n) \in[0, \bar{c}] \times[0,1]$. Then for any $n \in(0,1)$ we have $g(n)=\epsilon h^{\prime}(n)$ by A.7) and (3.8); thus (3.8) holds if and only if $n \in\left\{\eta_{1}, \ldots, \eta_{m}\right\}$. It remains to show that $u$ satisfies Assumption 2.2 for some $\epsilon \in \mathbb{R}$.

Recalling A.9 and A.12, we see that if $\epsilon=0$, then $u_{1}(c, n)>0, u_{2}(c, n)>$ 0 , and the Hessian matrix of $u$ at $(c, n)$ is negative definite for all $(c, n) \in$ $[0, \bar{c}] \times[0,1]$. Since these properties are preserved for $\epsilon$ sufficiently close to 0 , it follows that $u$ satisfies Assumption 2.2 for such $\epsilon$, as desired.

\section{A.7 Proof of Proposition 4.4}

Let $\bar{c}$ and $\phi$ be as in the proof of Proposition 4.2. Let $\epsilon>0$. Suppose that $u(c, n)=\phi(c, n)-\epsilon n$ for all $(c, n) \in[0, \bar{c}] \times[0,1]$. It is easy to see that $u$ satisfies Assumption 2.2. By (A.7) and (3.8) we have $g(n)=-\epsilon$ for all $n \in(0,1)$. Hence there is no interior steady state by Lemma 3.2 .

\section{References}

Barro J.R., and Salai-i-Martin, X., 2004, Economic Growth, Second Edition. MIT Press, Cambridge, Massachusetts.

Bond, E.W., Iwasa, K., Nishimura, K., 2012, The dynamic Hecksher-Ohlin model: a diagrammatic analysis, International Journal of Economic Theory $8,197-211$.

Bond, E.W., Iwasa, K., Nishimura, K., 2014, Dynamic two-country HecksherOhlin model with externality, International Journal of Economic Theory $10,53-74$. 
Brock, W.A., Mirman, L.J., 1972, Optimal economic growth and uncertainty: the discounted case, Journal of Economic Theory 4, 479-513.

Cai, Y., Kamihigashi, T., Stachurski, J., 2013, Stochastic optimal growth with risky labor supply, Journal of Mathematical Economics, forthcoming.

Cass, D., 1965, Optimum growth in an aggregative model of capital accumulation, Review of Economic Studies 32, 233-240.

Christiano, L.J., Eichenbaum, M., 1992, Current real-business-cycles theories and aggregate labor-market fluctuations," American Economic Review $82,430-450$.

De Hek, P.A., 1998, An aggregative model of capital accumulation with leisuredependent utility, Journal of Economic Dynamics and Control 23, 255276.

Diamond, P., 1965, National debt in a neoclassical growth model, American Economic Review 55, 1126-1150.

Galor, O., Ryder, H.E., 1989, Existence, uniqueness, and stability of equilibrium in an overlapping-generations model with productive capital, Journal of Economic Theory 49, 360-375.

Kamihigashi, T., 2005, Necessity of the transversality condition for stochastic models with bounded or CRRA utility, Journal of Economic Dynamics and Control 29, 1313-1329.

King, R.G., Plosser, C.I., Rebelo, S.T., 1988, Production, growth and business cycles: I. The basic neoclassical model, Journal of Monetary Economics $21,195-232$.

Koopmans, T.C., 1965, On the concept of optimal economic growth," in The Econometric Approach to Development Planning. Amsterdam: NorthHolland, pp. 225-300.

Kurz, M., 1968, Optimal economic growth and wealth effects, International Economic Review 9, 348-357.

Le Van, C., Nguyen, M.-H., Vailakis, Y., 2007, Equilibrium dynamics in an aggregative model of capital accumulation with heterogeneous agents and elastic labor, Journal of Mathematical Economics 43, 287-317.

Yano, M., 1999, Stability and turnpike theorems in dynamic competitive equilibrium, Japanese Economic Review 50, 398-421.

Yano, M., 2012, The von Neumann-McKenzie facet and the Jones duality theorem in two-sector optimal growth, International Journal of Economic Theory 8, 213-226. 